\title{
Development of a clinically useful tool for Prakriti assessment
}

\author{
Research Article
}

\section{Abhilash M1*, Sudhikumar K B²}

1. Assistant Professor, Department of Kriya Sharir, Government Ayurveda College, Tripunithura, Kerala, 2. Professor, Kerala University of Health Sciences, School of Research in Ayurveda, Tripunithura, Kerala.

\begin{abstract}
Introduction: Prakriti assessment is of fundamental importance for research and standardization of clinical practice. The available tools for prakriti assessment are not intended to be used in a clinical setting, which demands a short and clinically flexible one. Methods: 3 selected tools were compared with a newly developed questionnaire. These were administered first in 100 healthy volunteers. Agreement analysis between these tools were done. The validation process was completed by running the new tool together with TNMC questionnaire in 150 more individuals who have some doshavriddhi. The results were discussed in an FGD involving clinicians and faculties. Results: The new tool has shown fair agreement with Ayusoft (kappa 0.434 and Spearman correlation 0.506) and TNMC (kappa 0.429 and Spearman correlation 0.454) questionnaires. And it showed week agreement with selfassessment tool (kappa 0.214 and Spearman correlation 0.407). Meanwhile self-assessment tool has poor agreement with both Ayusoft (kappa 0.172 and Spearman correlation 0.279) and TNMC (kappa 0.175 and Spearman correlation 0.244). Reliability was tested in a total of 250 individuals and a Cronbach's alpha of 0.524 was obtained. Factor analysis was also done. In this total dataset, the new tool showed better agreement with TNMC questionnaire (kappa 0.581 and Spearman correlation 0.442). Conclusion: These results show that the new tool has potency to be run in large scale to study more variability among patients. This will add to the standardization of Ayurvedic diagnostic, prognostic and therapeutic fields.
\end{abstract}

Key Words: Prakriti, Tool, Agreement Analysis, Clinical assessment, Validity, Reliability.

\section{Introduction}

Understanding and assessment of Prakriti is inevitable part of Ayurvedic theory, education and practice. Also, the standardization of diagnostic, therapeutic and prophylactic sectors of Ayurveda demand the use of prakriti assessment and application to near perfection for extracting the maximum output of Ayurvedic management strategies. For this purpose, the need of the hour is a simple but powerful tool for prakriti assessment, which has maximum clinical efficiency. Hence this study was planned to device a new tool for prakriti assessment which renders valid and reliable result while assessing the prakriti and also can be used in various diseases. Then only, the actual purpose of prakriti assessment and subsequent decision making in Ayurvedic parlance can be achieved. The core Ayurvedic perspective of personalized medicine can be thus made fruitful by extending the assessment of individual characteristics contributed by the different guna factors and then negating the dosha variations created by the co-existing disease.

\section{* Corresponding Author:}

\section{Abhilash M}

Assistant Professor, Department of Kriya Sharir,

Government Ayurveda College,

Tripunithura -682301,

Kerala, India

Email Id: abhilash@ayurvedacollege.ac.in
Many works have been done and articles been published on the principles and practice of prakriti assessment. On reviewing them, we can experience the scope and utility of this area. Various tools in the form of questionnaires and softwares have been tested for their reliability and validity. Reliability is of prime importance and poor to substantial levels of reliability have been recorded for prakriti assessment. (1) Three questionnaires having different answering options were having good test-retest reliability according to numerical scores, but highly variable reliability according to discrete Ayurveda diagnosis. Internal consistency pertaining to individual constitutions within one questionnaire was poor for all three primary doshas, but especially for kapha. (2)

Several extensive works have been done in the area of standardization and assessment of prakriti. TRISUTRA consortium initiative is an integrative approach involving identification of molecular correlates of Prakriti as predictive and prognostic markers of disease as well as therapeutic response. (3) A comprehensive prakriti assessment model has been developed by CCRAS (Central Council for Research in Ayurvedic Sciences) and is being used for large scale testing in healthy volunteers. (4) Each question or parameter in a tool is important from a holistic point of view and ultimately it can contribute to strength or weakness of the tool. Since the Prakriti-based research work is still in its infantile stage, the evaluation of the full questionnaire/tool that they used to assess Prakriti 
along with the weightage they assigned to each item is important and has to be incorporated in the publications. (5)

Several genomic studies have also been conducted coupled with prakriti assessment. Principal component analysis (PCA) of 52 single nucleotide polymorphisms (SNPs) classified 262 individuals into their respective groups (Vata, Pitta and Kapha) irrespective of their ancestry, which represent its power in categorization. It was further validated using findings with 297 Indian population samples with known ancestry. (6) Exploration of the association between various Prakriti subtypes and other biological variables influencing a target phenotype is another promising area. Eventually, this may help in establishing the concept of "personalized medicine" in Ayurveda in its true sense. (7) Several Artificial Intelligence (AI) techniques are also being employed here. The experimental result shows that ensemble learning methods clearly surpasses conventional methods indicating that advances in boosting algorithms could give machine learning a leading future. (8) Thus, if conceptually and practically efficient tool is available for prakriti assessment, machine learning can be employed for majority of further works.

From theory to practice, it needs some rethinking in the application level. With an emphasis on Ayurvedic practice in Delhi Government Health Institutions, a descriptive study was conducted and it has highlighted the need to apply the patient-centric approach, which is the cornerstone of the Ayurvedic medical system. (9) The real strengths of Ayurveda are in the areas of health promotion, preventive, predictive and personalized medicine and they should be focussed on, instead of the present emphasis on therapeutics seems similar to practice of modern medicine. (10)

Still, the assessment of individual variations in the form of prakriti is difficult when compared to assessment of disease variations in the form of vikriti. A diagnostic reliability study of 30 patients and 4 Ayurvedic experts was conducted, nested in a randomized controlled trial. Patients were diagnosed in a sequential order by all experts utilizing a semi structured patient history form. A nominal group technique as consensus procedure was performed to reach agreement on the items to be diagnosed. While high percentages of agreement for main diagnostic entities and the final Ayurveda diagnosis (95\% consensus agreement on main diagnosis) could be observed, this was not reflected in the corresponding kappa values, which largely yielded fair-to-poor interrater agreement kappas for central diagnostic aspects such as prakriti and agni (k values between 0 and 0.4 ). Notably, agreement on disease-related entities was better than that on constitutional entities. (11) Another study showed the diabetic Vata Prakriti is a genetically susceptible group as it has a tendency to get affected by increased DNA damage, which could help in creating personalized management of diabetes among individual Prakriti. (12) There is a big area of scope for collaborative research between Ayurveda and other traditional medicines of Asia to enhance and streamline their inherent strengths of personalised medicine. (13)

A newly devised tool was good in reaching a consensus in reference to Vata and Pitta expressions, whereas it is notable to make a convincing correlation between observations made for Kapha group. Besides indicating the deficits related to the construct of the tool under study, it also indicates the intricate complexity associated with observations made in reference to Kapha features compared to Vata and Pitta. So, Kapha features are required to be designed more carefully to make their better appreciation by every observer, and therefore to reach a better agreement. (14) People with Kapha as the most dominant dosha showed a tendency to have either a higher parasympathetic activity or a lower sympathetic activity with respect to their cardiovascular reactivity in comparison to the individuals with Pitta or Vata as the most dominant Dosha. (15) The intrinsic activities of doshas have also been studied. The function of Ranjaka, Bhrajaka and Sadhaka Pitta was found better in Pitta Prakriti individuals followed by Kapha Prakriti and least in Vata Prakriti individuals. As mean values of hemoglobin, Short Term Memory and Long-Term Memory were highest in Pitta Prakriti and lowest in Vata Prakriti individuals. (16)

Prakriti assessment performed intuitively by the ayurvedic physician was having good correlation with Ayusoft measurements, thereby providing an effective and quantitative instrument to assess the prakriti of individuals. (17) Thus, the clinical adaptation of prakriti assessment using the understanding of tridosha theory has to be considered seriously while moving further to the area of personalized medicine. (18-22)

\section{Methodology}

The primary objectives of this study were,

- To develop a valid and reliable tool for prakriti assessment.

- To determine the prakriti of healthy volunteers and patients.

Secondary objective was,

- To provide database for the development of a clinical friendly software to determine prakriti.

The main drawback of the available questionnaires and softwares is that all of them are time consuming and hence cannot be administered in a clinical setting. Also, they do not consider the pathological changes in the assessment parameters so that they are rendered defective to be used in a clinical condition. Hence, a new tool developed based on the practicality and clinical friendliness was administered together with the other 3 tools namely,

1. Ayusoft - the software developed by C-DAC Pune. (23)

2. Questionnaire developed by Topiwala National Medical College (TNMC), Mumbai. (24)

3. Self-assessment questionnaire developed by Dr. Kishor Patwardhan et al. (25)

Assuming 8-10 parameters in the final scale, the initial sample size for agreement analysis was 
calculated as 100. Again, adding 3 dosha wise divisions to each item, the final sample size was calculated as 250 based upon the sample size criteria for health measurement scales. (26)

The new tool was developed taking the clinicalfriendly questions from the available questionnaires and analysing these questions in the light of gunas responsible. Another advantage of taking gunas into consideration is that it makes easy to find out the dwidoshaja traits which practically accounts for almost $95 \%$ of the population as per clinician's opinion.
Content validity of these questions were done taking opinion from 10 experts. (Table 1), The weightage based upon this expert opinion was added in the final analysis. Since the items are not new and they were only modifications of the available tools, Focus Group Discussion was deferred to the final step to summarize the findings and for clinical cross-checking. The tools were run in 100 healthy volunteers and the data obtained were statistically analysed. Agreement analysis between all these tools were done.

Table 1: Content Validity - Guna wise characteristics

\begin{tabular}{|c|c|c|c|c|c|c|}
\hline \multirow{2}{*}{ Characteristic } & \multicolumn{6}{|c|}{ Guna responsible } \\
\hline & Guru & Laghu & Snigdha & Rooksha & Ushna & Seeta \\
\hline \multicolumn{7}{|l|}{ Vataprakriti } \\
\hline Talkative & & 9 & & 5 & & \\
\hline Irrelevant talks & & 8 & & 8 & & 1 \\
\hline Appetite severe at times, diminished at times & & 5 & & 3 & & 5 \\
\hline Comprehension \& Memory - Grasps easily, forgets easily & & 6 & & 4 & & 1 \\
\hline Prefer sweet & & 6 & & 4 & & 1 \\
\hline Dry skin & & & & 10 & & 4 \\
\hline Round, lusterless eyes & & & & 6 & & 1 \\
\hline Wakes up from sleep intermittently & & 8 & & 4 & & \\
\hline Few friends & & 2 & & 5 & & 5 \\
\hline Prefers hot & & 1 & & 1 & & 8 \\
\hline Prefers sour & & 2 & & 3 & & 7 \\
\hline Prefers salt & & 3 & & 3 & & 6 \\
\hline \multicolumn{7}{|l|}{ Pittaprakriti } \\
\hline Speaks harshly when angry & & 2 & 1 & & 6 & \\
\hline Intolerance to hunger & & 3 & & & 9 & \\
\hline Becomes support for the dependents & & 1 & 5 & & 2 & \\
\hline Strong appetite and digestive capacity & & 4 & 3 & & 8 & \\
\hline Moderate sleep & & 4 & 2 & & 2 & \\
\hline Profuse sweating & & 4 & & & 7 & \\
\hline Eyes turns red easily & & 2 & & & 7 & \\
\hline Curious in new matters and ideas & & 2 & 5 & & 5 & \\
\hline Prefers cold & & 2 & & & 7 & \\
\hline Prefers sweet & & 6 & 1 & & 7 & \\
\hline Prefers bitter & & 4 & 1 & & 7 & \\
\hline \multicolumn{7}{|l|}{ Kaphaprakriti } \\
\hline Wide and elongated eyes & 4 & & 4 & & & 2 \\
\hline Generally reduced appetite & 2 & & 2 & & & 7 \\
\hline Hunger can be tolerated & 4 & & 2 & & & 4 \\
\hline Deep sleep & 5 & & 4 & & & 5 \\
\hline Grasps slowly but good memory & 6 & & 3 & & & 3 \\
\hline Smooth skin & 1 & & 7 & & & 2 \\
\hline Plenty of eye lashes & 4 & & 3 & & & 3 \\
\hline Relevant \& Soft spoken & 4 & & 6 & & & 3 \\
\hline Strong friendship & 3 & & 7 & & & 3 \\
\hline Moist skin & & & 6 & & & 1 \\
\hline Prefers hot & 2 & & & & & 8 \\
\hline Prefers bitter & 6 & & 5 & & & 3 \\
\hline Prefers pungent & 5 & & 7 & & & 5 \\
\hline Prefers astringent & 1 & & 8 & & & 2 \\
\hline
\end{tabular}

Again, the new clinical friendly tool developed for prakriti assessment was run in 150 more individuals who have some doshavriddhi, together with TNMC questionnaire. Due to Covid19 pandemic, this assessment could be done as online only and hence the questionnaire was converted into google forms.

https://docs.google.com/forms/d/e/ 1 F A I p Q L S di Vw D v 6 P n 045 M c L Q i F 4 r A OER9P2KOTtORUzWocD5WFa-VjQ/view form? usp=sf_link
The data obtained were tabulated and the final score for the new tool was attributed based upon the guna factors common in the dwidoshaja status; so that it directly renders the dwidoshaja results. The individual scores of vata, pitta and kapha as well as that of vatapitta, vatakapha and pittakapha were noted. Considering the scores from these calculations, final diagnosis of prakriti was done.

Reliability was tested in this total of 250 individuals and the results were summarised. These 
results and their interpretations were discussed in an FGD to streamline the clinical needs and to increase the usefulness of the tool.

\section{Results}

Most of the participants of the study were students and hence belong to the group of 20-30 age group (Fig. 1). Since the study was conducted in Govt Ayurveda College, Tripunithura and mainly among the UG and PG students, most of the participants (85.6\%) were females.
The new tool has shown fair agreement with Ayusoft (kappa 0.434 and Spearman correlation 0.506) and TNMC (kappa 0.429 and Spearman correlation $0.454)$ questionnaires. And it showed week agreement with Kishor Patwardhan's tool (kappa 0.214 and Spearman correlation 0.407) (Table 2). Meanwhile Kishor Patwardhan's tool has poor agreement with both Ayusoft (kappa 0.172 and Spearman correlation 0.279) and TNMC (kappa 0.175 and Spearman correlation 0.244). Ayusoft and TNMC have the best agreement between them (kappa 0.576 and Spearman correlation 0.516) (Table 3).

Figure 1. Age wise distribution of participants. X-axis - Age; Y-axis - Number of participants

Age

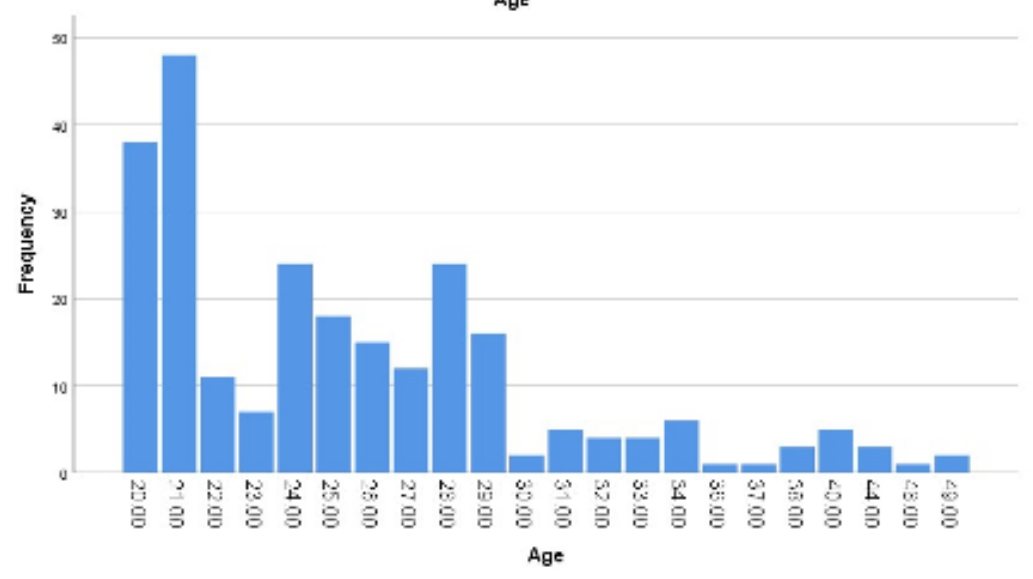

Table 2. Agreement between new tool and other tools

\begin{tabular}{|c|c|c|c|c|c|c|c|c|}
\hline & \multicolumn{7}{|c|}{ Prakriti Ayusoft / TNMC / Self } \\
\hline & & VP & VK & PK & V & $\mathbf{P}$ & $\mathbf{K}$ & $\mathbf{S}$ \\
\hline \multirow{5}{*}{$\begin{array}{c}\text { New } \\
\text { Prakriti }\end{array}$} & VP & $17 / 15 / 12$ & $5 / 5 / 10$ & $1 / 1 / 3$ & $2 / 3 / 0$ & $0 / 1 / 0$ & $1 / 1 / 0$ & $0 / 0 / 1$ \\
\hline & VK & $0 / 0 / 1$ & $1 / 1 / 0$ & $0 / 0 / 0$ & $0 / 0 / 0$ & $0 / 0 / 0$ & $0 / 0 / 0$ & $0 / 0 / 0$ \\
\hline & PK & $9 / 3 / 8$ & $5 / 9 / 18$ & $51 / 52 / 37$ & $0 / 1 / 2$ & $2 / 3 / 0$ & $2 / 2 / 4$ & $2 / 1 / 2$ \\
\hline & $\mathbf{P}$ & $0 / 0 / 1$ & $0 / 0 / 0$ & $1 / 1 / 0$ & $0 / 0 / 0$ & $0 / 0 / 0$ & $0 / 0 / 0$ & $0 / 0 / 0$ \\
\hline & $\mathbf{K}$ & $0 / 0 / 0$ & $0 / 0 / 0$ & $1 / 1 / 1$ & $0 / 0 / 0$ & $0 / 0 / 0$ & $0 / 0 / 0$ & $0 / 0 / 0$ \\
\hline Total & $26 / 18 / 22$ & $11 / 15 / 28$ & $54 / 55 / 41$ & $2 / 4 / 2$ & $2 / 4 / 0$ & $3 / 3 / 4$ & $2 / 1 / 3$ & \\
\hline \multicolumn{2}{|c|}{ Spearman Correlation } & \multicolumn{7}{|c|}{$0.506 / 0.454 / 0.407$} \\
\hline \multicolumn{2}{|c|}{ Kappa } & \multicolumn{7}{|c|}{$0.434 / 0.429 / 0.214$} \\
\hline
\end{tabular}

Table 3. Agreement between other tools

Prakrit Ayusoft vs Prakriti self-assessment

\begin{tabular}{|c|c|c|c|c|c|c|c|c|}
\hline \multicolumn{9}{|c|}{ Prakrit Ayusoft vs Prakriti self-assessment } \\
\hline & & & & & & & & \\
\hline & & VP & VK & PK & $\mathrm{V}$ & K & $\mathrm{S}$ & Total \\
\hline \multirow{7}{*}{ Prakriti ayusoft } & VP & 9 & 11 & 4 & 0 & 0 & 2 & 26 \\
\hline & VK & 4 & 2 & 4 & 1 & 0 & 0 & 11 \\
\hline & PK & 6 & 14 & 30 & 1 & 3 & 0 & 54 \\
\hline & $\mathrm{V}$ & 2 & 0 & 0 & 0 & 0 & 0 & 2 \\
\hline & $\mathrm{P}$ & 1 & 1 & 0 & 0 & 0 & 0 & 2 \\
\hline & $\mathrm{K}$ & 0 & 0 & 2 & 0 & 1 & 0 & 3 \\
\hline & $\mathrm{S}$ & 0 & 0 & 1 & 0 & 0 & 1 & 2 \\
\hline \multicolumn{2}{|l|}{ Total } & 22 & 28 & 41 & 2 & 4 & 3 & 100 \\
\hline \multicolumn{9}{|c|}{ Prakrit TNMC vs Prakriti self-assessment } \\
\hline & & \multicolumn{6}{|c|}{ Prakriti self } & \multirow{2}{*}{ Total } \\
\hline & & VP & VK & PK & $\mathrm{V}$ & K & $\mathrm{S}$ & \\
\hline \multirow{7}{*}{ Prakriti TNMC } & VP & 6 & 8 & 3 & 0 & 0 & 1 & 18 \\
\hline & VK & 6 & 4 & 3 & 2 & 0 & 0 & 15 \\
\hline & PK & 6 & 14 & 31 & 0 & 3 & 1 & 55 \\
\hline & $\mathrm{V}$ & 2 & 0 & 2 & 0 & 0 & 0 & 4 \\
\hline & $\mathrm{P}$ & 2 & 1 & 1 & 0 & 0 & 0 & 4 \\
\hline & $\mathrm{K}$ & 0 & 1 & 1 & 0 & 1 & 0 & 3 \\
\hline & $\mathrm{S}$ & 0 & 0 & 0 & 0 & 0 & 1 & 1 \\
\hline Total & & 22 & 28 & 41 & 2 & 4 & 3 & 100 \\
\hline
\end{tabular}




\section{Prakrit TNMC vs Prakriti Ayusoft}

\begin{tabular}{|c|c|c|c|c|c|c|c|c|c|}
\hline & \multicolumn{6}{|c|}{ Prakriti ayusoft } & & \multirow{2}{*}{ Total } \\
\hline & & VP & VK & PK & $\mathrm{V}$ & $\mathrm{P}$ & $\mathrm{K}$ & $\mathrm{S}$ & \\
\hline \multirow{8}{*}{ Prakriti TNMC } & VP & 15 & 2 & 1 & 0 & 0 & 0 & 0 & 18 \\
\hline & VK & 1 & 6 & 6 & 0 & 1 & 1 & 0 & 15 \\
\hline & PK & 6 & 3 & 46 & 0 & 0 & 0 & 0 & 55 \\
\hline & $\mathrm{V}$ & 2 & 0 & 0 & 2 & 0 & 0 & 0 & 4 \\
\hline & $\mathrm{P}$ & 1 & 0 & 1 & 0 & 1 & 0 & 1 & 4 \\
\hline & $\mathrm{K}$ & 1 & 0 & 0 & 0 & 0 & 2 & 0 & 3 \\
\hline & $\mathrm{S}$ & 0 & 0 & 0 & 0 & 0 & 0 & 1 & 1 \\
\hline & Total & 26 & 11 & 54 & 2 & 2 & 3 & 2 & 100 \\
\hline
\end{tabular}

\section{Face validity and content validity}

The face validity of the tool was directly tested form the clinicians who are used to look for prakriti wise differences in patients. The content validity of the new tool was determined by getting feedback from 10 experts regarding the guna wise weightage for the individual items in the tool.

\section{Criterion validity}

Criterion validity was obtained by comparing with other standard tools like Ayusoft, TNMC and selfassessment questionnaire. The fair agreement with Ayusoft and TNMC shows that it has good criterion validity.

\section{Construct validity}

Feedback was taken from the clinicians regarding the factors under consideration for prakriti assessment and the impact of various gunas on them
(Fig. 2). Inputs from FGD were also used for this purpose. Further, reliability testing separately based upon individual doshas were also done. (Table 4)

Figure 2. Construct of prakriti and its components.

Prakriti divided into 3 phases - evolution, expression and influence of disease

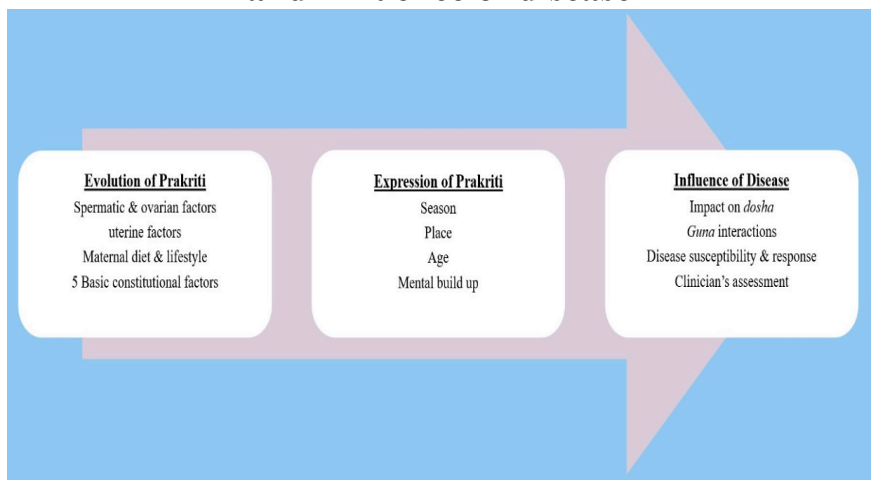

Table 4. Reliability testing based upon individual doshas Item Statistics

\begin{tabular}{|c|c|c|c|c|}
\hline \multicolumn{4}{|c|}{ Item Statistics } & \multirow{2}{*}{ Cronoacn's Alpna } \\
\hline Kapha & Mean & Std. Deviation & $\mathbf{N}$ & \\
\hline Smooth skin & 2.10 & 0.877 & 250 & 0.603 \\
\hline Eye wide & 1.88 & 0.917 & 250 & \\
\hline Soft spoken & 2.19 & 0.878 & 250 & \\
\hline Appetite less & 1.74 & 0.902 & 250 & \\
\hline Sleep sound & 2.02 & 0.942 & 250 & \\
\hline Memory good & 1.92 & 0.877 & 250 & \\
\hline Astringent & 1.39 & 1.267 & 250 & \\
\hline Strong friend & 2.08 & 0.958 & 250 & \\
\hline \multicolumn{5}{|l|}{ Pitta } \\
\hline Sweating & 1.86 & 0.905 & 250 & 0.519 \\
\hline Eye red & 1.57 & 0.829 & 250 & \\
\hline Talk rough & 1.74 & 0.905 & 250 & \\
\hline Appetite strong & 1.84 & 0.920 & 250 & \\
\hline Sleep moderate & 1.86 & 0.913 & 250 & \\
\hline Innovative & 2.09 & 0.942 & 250 & \\
\hline Sweet & 1.60 & 1.138 & 250 & \\
\hline Bitter & 1.15 & 1.258 & 250 & \\
\hline Friend support & 2.28 & 0.906 & 250 & \\
\hline \multicolumn{5}{|l|}{ Vata } \\
\hline Dry skin & 1.71 & 0.840 & 250 & 0.540 \\
\hline Eye lustreless & 1.76 & 0.882 & 250 & \\
\hline Talkative & 1.79 & 0.887 & 250 & \\
\hline Appetite varied & 1.79 & 0.905 & 250 & \\
\hline Sleep disturbed & 1.56 & 0.830 & 250 & \\
\hline Easy grasp and forget & 1.86 & 0.929 & 250 & \\
\hline Hot & 1.55 & 1.165 & 250 & \\
\hline Sweet & 1.60 & 1.138 & 250 & \\
\hline Few friend & 1.42 & 0.747 & 250 & \\
\hline
\end{tabular}




\section{Factor Analysis and Internal Consistency}

Bartlett's test of sphericity shows significant variation among the recorded data with degree of freedom 300 . Still, KMO measure of 0.462 shows it has to be tested in a larger sample. Factor analysis was also done. 7 factors were identified in the scree plot (Fig. 3). Factor loading also supports this finding by showing the dosha wise as well as dwidoshaja characteristics loaded under different factors, even though it has to be confirmed with a larger sample. (Table 5).

Figure 3 - Scree Plot

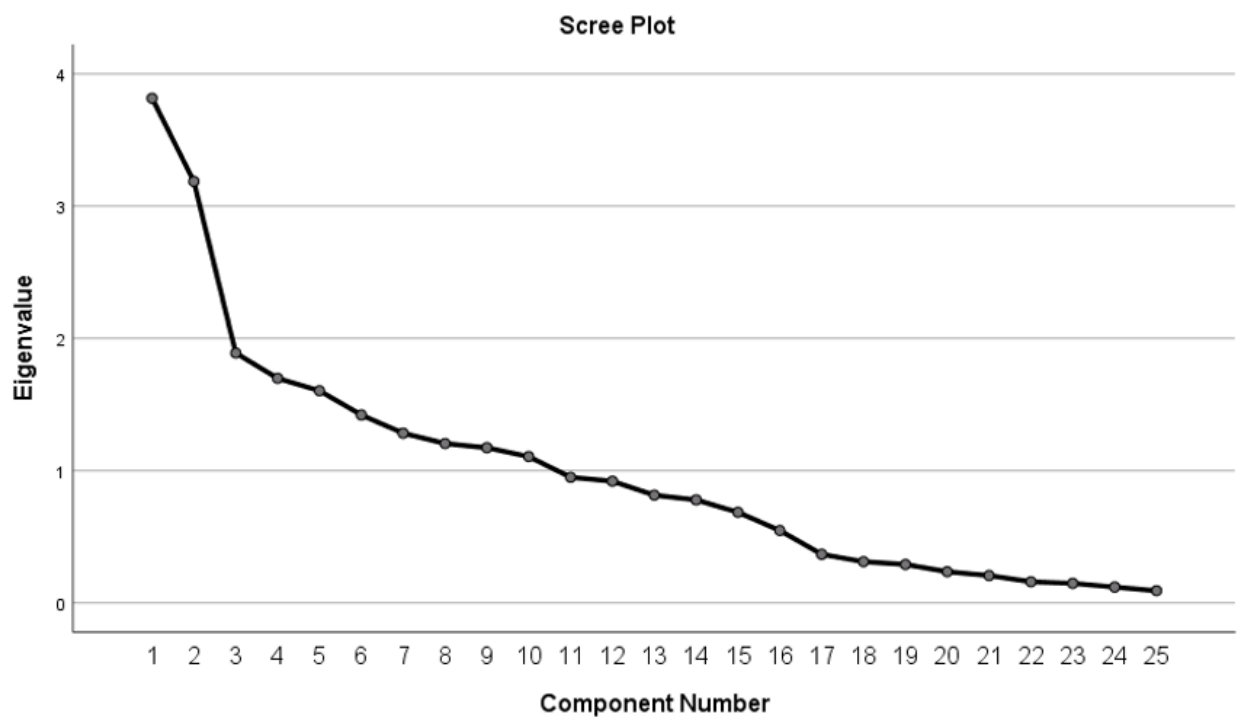

$\mathrm{X}$-axis - component number; Y-axis - eigen value; 7 factors clearly identified

Table 5. Factor loading Rotated Component Matrix ${ }^{a}$

\begin{tabular}{|c|c|c|c|c|c|c|c|c|c|c|}
\hline \multicolumn{11}{|c|}{ Rotated Component Matrix ${ }^{\mathrm{a}}$} \\
\hline & & & & & Com & nent & & & & \\
\hline & 1 & 2 & 3 & 4 & 5 & 6 & 7 & 8 & 9 & 10 \\
\hline Dry skin & 0.026 & 0.129 & -0.179 & 0.074 & -0.019 & -0.136 & 0.081 & -0.042 & -0.889 & -0.031 \\
\hline Sweating & 0.045 & 0.286 & -0.157 & 0.148 & 0.037 & -0.294 & 0.195 & -0.144 & 0.556 & 0.034 \\
\hline Smooth skin & 0.074 & 0.150 & 0.446 & -0.079 & 0.110 & 0.366 & -0.114 & 0.252 & 0.426 & 0.218 \\
\hline Eye lustreless & 0.052 & -0.052 & 0.047 & -0.068 & -0.167 & -0.766 & 0.122 & -0.037 & -0.103 & 0.306 \\
\hline Eye red & -0.097 & 0.575 & -0.162 & 0.172 & 0.065 & -0.124 & -0.367 & 0.143 & 0.254 & -0.173 \\
\hline Eye wide & 0.107 & -0.027 & 0.080 & 0.043 & -0.029 & 0.844 & 0.148 & -0.127 & -0.055 & 0.154 \\
\hline Talkative & -0.041 & 0.186 & -0.713 & -0.039 & 0.030 & 0.039 & 0.099 & 0.024 & -0.119 & 0.300 \\
\hline Talk rough & 0.135 & 0.598 & 0.022 & 0.349 & 0.051 & -0.079 & 0.253 & 0.013 & 0.077 & 0.189 \\
\hline Soft spoken & 0.112 & 0.019 & 0.818 & -0.066 & 0.094 & 0.089 & -0.174 & 0.000 & 0.005 & -0.004 \\
\hline Appetite varied & -0.099 & 0.067 & -0.387 & 0.153 & -0.616 & -0.007 & -0.141 & 0.286 & 0.013 & 0.261 \\
\hline Appetite strong & 0.070 & 0.145 & 0.115 & -0.028 & 0.205 & 0.066 & 0.025 & -0.892 & 0.007 & -0.012 \\
\hline Appetite less & 0.100 & 0.255 & 0.341 & 0.075 & 0.438 & -0.021 & 0.165 & 0.639 & 0.011 & 0.041 \\
\hline Sleep disturbed & 0.165 & 0.448 & -0.341 & 0.048 & -0.116 & 0.134 & 0.219 & 0.216 & 0.160 & -0.312 \\
\hline Sleep moderate & 0.078 & 0.135 & 0.145 & 0.869 & 0.045 & 0.031 & 0.061 & -0.010 & -0.044 & 0.269 \\
\hline Sleep sound & -0.047 & -0.010 & 0.260 & -0.767 & 0.112 & -0.094 & -0.077 & -0.099 & -0.031 & 0.357 \\
\hline Easy grasp and forget & 0.036 & 0.113 & -0.092 & 0.220 & 0.032 & -0.001 & 0.806 & 0.090 & 0.058 & 0.111 \\
\hline Innovative & 0.036 & 0.729 & -0.091 & -0.119 & 0.100 & 0.083 & 0.035 & -0.150 & -0.099 & 0.179 \\
\hline Memory good & 0.126 & -0.033 & 0.414 & 0.107 & 0.131 & -0.017 & -0.662 & 0.066 & 0.082 & 0.125 \\
\hline Hot & -0.867 & 0.057 & -0.107 & -0.014 & -0.040 & -0.088 & 0.058 & -0.002 & -0.039 & 0.113 \\
\hline Sweet & -0.895 & 0.022 & -0.060 & -0.045 & -0.051 & 0.038 & 0.029 & 0.033 & -0.013 & 0.038 \\
\hline Bitter & 0.517 & 0.542 & 0.221 & 0.159 & -0.034 & 0.047 & 0.078 & 0.053 & -0.076 & 0.243 \\
\hline astringent & 0.747 & 0.333 & -0.062 & 0.035 & 0.180 & 0.015 & 0.073 & -0.008 & -0.032 & 0.169 \\
\hline Few friend & 0.015 & 0.164 & -0.191 & 0.041 & 0.037 & -0.042 & 0.046 & 0.045 & 0.093 & 0.730 \\
\hline Friend support & 0.031 & 0.444 & 0.353 & 0.115 & -0.628 & -0.019 & 0.279 & -0.023 & -0.086 & -0.158 \\
\hline Strong friend & 0.192 & 0.270 & 0.073 & 0.077 & 0.724 & 0.128 & -0.064 & -0.002 & 0.038 & 0.111 \\
\hline
\end{tabular}

Extraction Method: Principal Component Analysis.

Rotation Method: Varimax with Kaiser Normalization.

a. Rotation converged in 13 iterations.

Reliability was tested in this total of 250 individuals and a Cronbach's alpha of 0.524 was obtained (Table 6). In this total dataset, the new tool showed better agreement with TNMC questionnaire (kappa 0.581 and Spearman correlation 0.442) (Table 7). The lesser scores of kappa and KMO coefficient can be attributed to the construct of Prakriti, which has many dimensions and the aspects which are clinically relevant only being considered in this study. 


\section{Table 6. Internal Consistency of the new tool \\ Item-Total Statistics}

\begin{tabular}{|c|c|c|c|c|c|}
\hline & $\begin{array}{c}\text { Scale Mean if Item } \\
\text { Deleted }\end{array}$ & $\begin{array}{l}\text { Scale Variance if } \\
\text { Item Deleted }\end{array}$ & $\begin{array}{l}\text { Corrected Item } \\
\text { Total Correlation }\end{array}$ & $\begin{array}{l}\text { Squared Multiple } \\
\text { Correlation }\end{array}$ & $\begin{array}{l}\text { Cronbach's Alpha } \\
\text { if Item Deleted }\end{array}$ \\
\hline Dry skin & 45.70 & 47.187 & -0.028 & 0.583 & 0.537 \\
\hline Sweating & 45.55 & 44.418 & 0.193 & 0.463 & 0.508 \\
\hline Smooth skin & 45.32 & 44.403 & 0.205 & 0.643 & 0.506 \\
\hline Eye lustreless & 45.65 & 47.084 & -0.024 & 0.487 & 0.537 \\
\hline Eye red & 45.84 & 44.746 & 0.194 & 0.385 & 0.508 \\
\hline Eye wide & 45.53 & 46.363 & 0.030 & 0.490 & 0.531 \\
\hline Talkative & 45.62 & 46.205 & 0.048 & 0.583 & 0.528 \\
\hline Talk rough & 45.67 & 40.409 & 0.551 & 0.601 & 0.455 \\
\hline Soft spoken & 45.22 & 45.949 & 0.072 & 0.684 & 0.524 \\
\hline Appetite varied & 45.63 & 47.291 & -0.043 & 0.696 & 0.540 \\
\hline Appetite strong & 45.56 & 46.425 & 0.024 & 0.722 & 0.531 \\
\hline Appetite less & 45.67 & 43.649 & 0.261 & 0.733 & 0.498 \\
\hline Sleep disturbed & 45.85 & 45.006 & 0.168 & 0.598 & 0.512 \\
\hline Sleep moderate & 45.55 & 43.168 & 0.298 & 0.693 & 0.493 \\
\hline Sleep sound & 45.39 & 47.714 & -0.079 & 0.692 & 0.546 \\
\hline Easy grasp and forget & 45.55 & 44.192 & 0.204 & 0.586 & 0.506 \\
\hline Innovative & 45.31 & 41.135 & 0.460 & 0.592 & 0.467 \\
\hline Memory good & 45.49 & 46.904 & -0.008 & 0.600 & 0.535 \\
\hline Hot & 45.86 & 48.850 & -0.162 & 0.579 & 0.568 \\
\hline Sweet & 45.81 & 49.880 & -0.224 & 0.637 & 0.576 \\
\hline Bitter & 46.27 & 37.841 & 0.528 & 0.572 & 0.436 \\
\hline Astringent & 46.02 & 40.371 & 0.347 & 0.614 & 0.475 \\
\hline Few friend & 45.99 & 44.044 & 0.299 & 0.544 & 0.497 \\
\hline Friend support & 45.13 & 44.277 & 0.205 & 0.631 & 0.506 \\
\hline Strong friend & 45.33 & 42.583 & 0.326 & 0.678 & 0.487 \\
\hline Prakriti new & 44.76 & 45.258 & 0.090 & 0.391 & 0.523 \\
\hline
\end{tabular}

Table 7. Total Agreement between new tool and TNMC questionnaire Prakriti new * prakriti TNMC Crosstabulation

\begin{tabular}{|c|c|c|c|c|c|c|c|c|c|}
\hline \multicolumn{10}{|c|}{ Prakriti new * prakriti TNMC Crosstabulation } \\
\hline & & & & & & & & & \\
\hline & & \multicolumn{6}{|c|}{ Prakriti TNMC } & \multirow[b]{2}{*}{$\mathrm{S}$} & \multirow[t]{2}{*}{ Total } \\
\hline & & VP & VK & PK & $\mathrm{V}$ & $\mathrm{P}$ & $\mathrm{K}$ & & \\
\hline \multirow{6}{*}{ Prakriti new } & VP & 30 & 5 & 11 & 2 & 2 & 0 & 3 & 53 \\
\hline & VK & 1 & 5 & 1 & 0 & 0 & 0 & 0 & 7 \\
\hline & PK & 8 & 3 & 162 & 1 & 0 & 3 & 1 & 178 \\
\hline & $\mathrm{V}$ & 0 & 0 & 1 & 2 & 0 & 0 & 0 & 3 \\
\hline & $\mathrm{P}$ & 1 & 1 & 1 & 0 & 3 & 0 & 0 & 6 \\
\hline & $\mathrm{K}$ & 2 & 0 & 1 & 0 & 0 & 0 & 0 & 3 \\
\hline \multicolumn{2}{|c|}{ Total } & 42 & 14 & 177 & 5 & 5 & 3 & 4 & 250 \\
\hline
\end{tabular}

\section{Kappa 0.581}

\section{FGD findings}

A Focus Group Discussion was conducted among the clinicians and academicians (Fig. 4). The recording was transcribed and translated. After open coding, axial coding and selective coding, 4 themes were identified from the FGD. The general pattern of the practice of prakriti; the variations in understanding and assessment; the general uses in clinical practice as well as the issues in the clinical assessment of prakriti were highlighted in the FGD (Table 8). The characteristics added in the new tool were re-identified as the clinically relevant findings of prakriti from the FGD. The importance of identifying the dwidoshaja traits were also highlighted. (Fig. 5) 
Figure 4: Sociogram - FGD on clinical use of Prakriti assessment (18-11-2020)

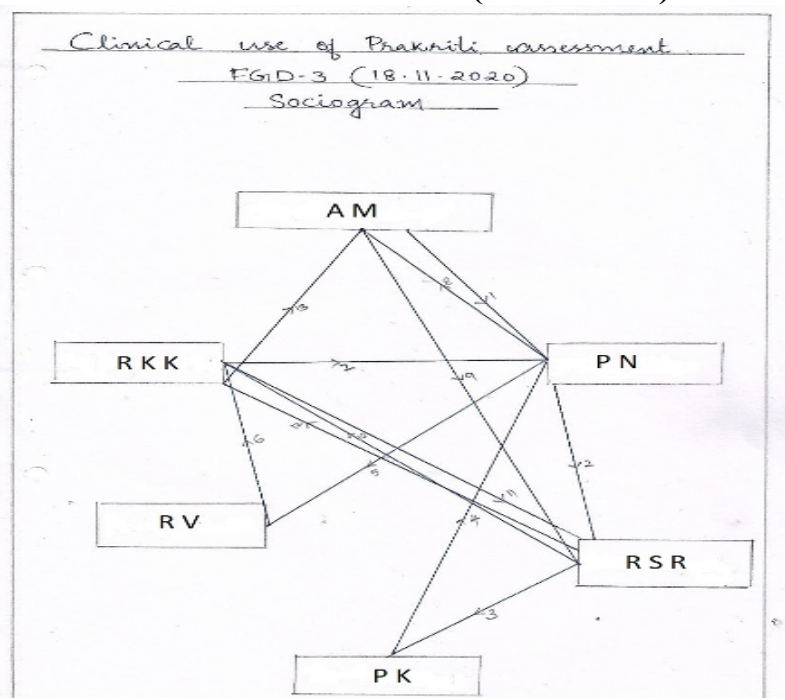

AM - Moderator; RKK - Participant 1; RV - Participant 2; PK - Participant 3; RSR - Participant 4; PN Participant 5

Table 8. Themes and subthemes in FGD

Themes

Practice

No systematic assessment; Rough assessment only

Confused with dosha assessment In understanding (physician's)

Sub-themes Key role of pitta and agni; hence pitta (VP, PK) and non-pitta

(VK) can be considered clinically

Indirectly from koshta

Direct observations of height, skin, eyes, satmya etc

Variation

Difference in opinion of Acharyas regarding the weightage

In assessment (weightage for dosha expression)

Patient might have forgotten their koshta condition because of chronicity of their disease.

There may be changes according to the vikriti.
Figure 5: The word cloud representation of discussions in FGD

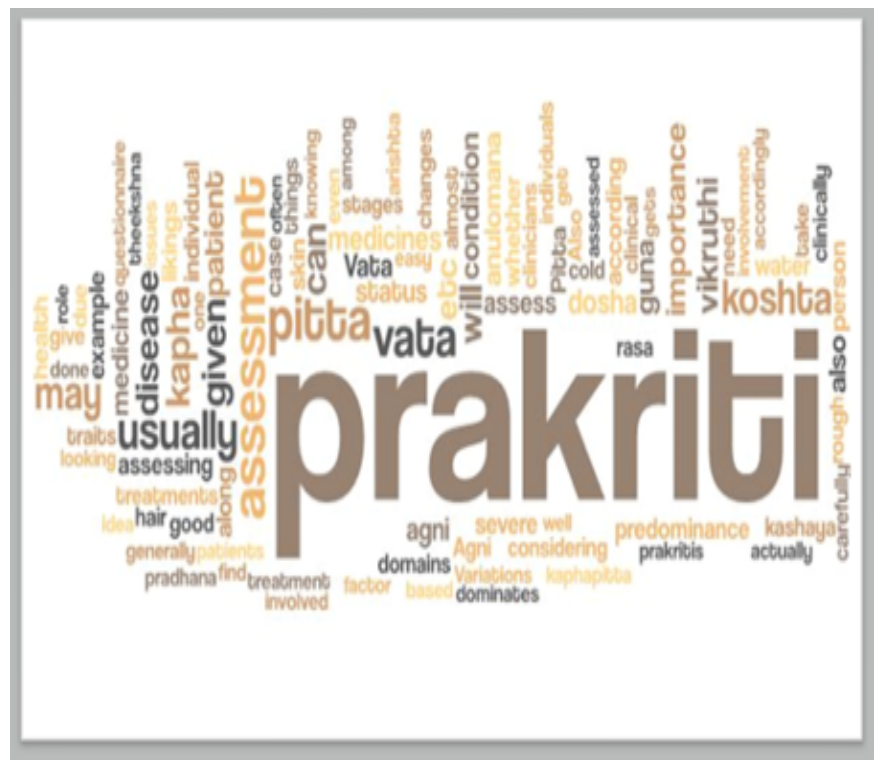

Uses

To know disease susceptibility

To determine prognosis

For choice of medicine and dosage

For choice of Pathya

For clinical feedback
Issues

Prakriti and vikriti demarcation

Long-standing vikriti can interfere with their prakriti.

Pitta is often missed

Unable to explain clinically

Failure to recall by patients

\section{Discussion}

This study findings indicate that prakriti assessment has to be incorporated as an integral part of clinical examination in Ayurveda to achieve the proclaimed status of personalized medicine. (18) The available tools do not have perfect agreement with each other. (2) Also, they cannot be directly subjected to clinical testing to find out whether they actually addressed the construct of prakriti or not. Under these circumstances, it can be highlighted that the new tool developed in this study can be the best option to be subjected to clinical testing in a big sample and to see whether it can withstand the internal variations in the organization and expression of prakriti.

The fair agreement of the new tool with Ayusoft and TNMC shows that it has good concurrent validity. The self-assessment tool developed by Kishor Patwardhan et al can be seen as differing from all the other tools, mainly due to the change in pattern of assessment. Still, compared to Ayusoft and TNMC, the new tool has better agreement with self-assessment tool, owing to its efficiency in recording the subject's own feelings also. Some of the parameters, which creates doubt in the minds of those filling the forms may have great influence in the final outcome of the assessment. (20-22) It can be conclusively stated that no such factors are considered in the new tool and thus it is very less susceptible to faulty assessment and result.

Another advantage of this tool is that it can be used as self-assessment tool as well as physician administered tool with slight changes in the outlook. Also, it can be easily converted into software or mobile application without much modifications. (8) It can be filled within 5-7 minutes in both physician administered and self-administered modes. The direct linking with the dominant guna of the combination of doshas makes the clinical adaptation to maximum efficiency, which can also be cross checked in different disease conditions. This was highlighted in the FGD and various links connecting the clinical importance of prakriti assessment were narrated.

\section{Conclusion}

These results show that the new tool has potency to be run in large scale to study more variability 
among patients suffering from different diseases and to test the treatment response accordingly to confirm the findings. This will add to the standardization of Ayurvedic diagnostic, prognostic and therapeutic fields.

\section{Limitations}

- The study was more focussed on the 20-30 age group since it was done mainly in healthy volunteers in Govt Ayurveda College, Tripunithura

- The second phase of administration of the tool in 150 doshavriddhi states could be done as online only due to the Covid19 pandemic.

\section{Suggestions}

- Follow up study can be planned taking specific disease conditions into consideration.

- Software development and mobile app designing can be done based upon this tool

- Using the electronic media, a data bank of prakriti characteristics and clinical features can be developed and further big data analysis can be planned.

\section{Acknowledgements}

The authors gratefully acknowledge the financial support from "Kerala University of Health Sciences" through the newly formed "School of Research in Ayurveda." The authors also acknowledge Late Dr. Prakash Mangalasseri, former Associate Professor, Dept of Kayachikitsa, VPSV Ayurveda College, Kottakkal, for being an inspirational force in the beginning of this work. The authors are thankful to Dr. Benil P.B., Associate Professor, Dept of Agadatantra, VPSV Ayurveda College, Kottakkal for guiding in the statistical analysis and interpretation. The authors gratefully acknowledge cooperation of all the volunteers who have participated in this study.

\section{Statement of Ethics}

IRB approval for the study was obtained from the institutional committee for ethics in Govt Ayurveda College, Tripunithura (Ref: 03/KS-PJ-KUHS/IEC/2018 dated 25-04-2018 and revalidated as 39-03/KS-PJKUHS/IEC/2019 dated 11-04-2019). An informed consent form expressing the consent to share their Prakriti details for the study was developed and it was translated into local language, Malayalam. The duly filled consent form was obtained from all the participants. Verbal consent was taken from the participants of online Focus Group Discussion on 'clinical use of Prakriti Assessment' conducted in google meet platform on 18-11-2020.

\section{Data availability statement}

All data generated or analyzed during this study are included in this article and its supplementary material files. Further enquiries can be directed to the corresponding author.

\section{Author disclosure statement} disclose

\section{Funding statement}

Rs. 1,00,000/- (Rupees One Lakh only) was sanctioned by Kerala University of Health Sciences and was utilised for the remuneration of Junior Research Fellow for 2 months, for purchase of Ayusoft full suite instrument and for other expenses as a part of the study.

\section{References}

1. Kurande V, Bilgrau AE, Waagepetersen R, Toft E, Prasad R. Interrater reliability of diagnostic methods in traditional Indian ayurvedic medicine. Evidence-Based Complementary and Alternative Medicine. 2013 Sep 26;2013. https://doi.org/ 10.1155/2013/658275

2. Dunlap C, Hanes D, Elder C, Nygaard C, Zwickey H. Reliability of self-reported constitutional questionnaires in Ayurveda diagnosis. Journal of Ayurveda and integrative medicine. 2017 Oct 1;8(4):257-62. https:// doi.org/10.1016/j.jaim.2017.04.011

3. Prasher B, Varma B, Kumar A, Khuntia BK, Pandey R, Narang A, Tiwari P, Kutum R, Guin D, Kukreti R, Dash D. Ayurgenomics for stratified medicine: TRISUTRA consortium initiative across ethnically and geographically diverse Indian populations. Journal of ethnopharmacology. 2017 Feb 2;197:274-93. https://doi.org/10.1016/j.jep.2016.07.063

4. Singh R Ota, Bharati, Srikanth N, Dhiman KS, Sharma L. Development of Standardized Prakriti Assessment Tool: An Overview of Ongoing CCRAS Initiatives. J Res Ayurvedic Sci 2017; 1(3):165-208

5. Bhalerao S, Patwardhan K. Prakriti-based research: Good reporting practices. Journal of Ayurveda and integrative medicine. $2016 \mathrm{Mar}$ 1;7(1):69-72. https://doi.org/10.1016/ j.jaim.2015.08.002

6. Govindaraj, P., Nizamuddin, S., Sharath, A. et al. Genome-wide analysis correlates Ayurveda Prakriti. Sci Rep 5, 15786 (2015). https://doi.org/ 10.1038/srep15786

7. Thaker SJ, Gandhe PP, Godbole CJ, Bendkhale SR, Mali NB, Thatte UM, Gogtay NJ. A prospective study to assess the association between genotype, phenotype and Prakriti in individuals on phenytoin monotherapy. Journal of Ayurveda and integrative medicine. 2017 Jan 1;8(1):37-41. https://doi.org/10.1016/ j.jaim.2016.12.001

8. V. Madaan and A. Goyal, "Predicting AyurvedaBased Constituent Balancing in Human Body Using Machine Learning Methods," in IEEE 
Access, vol. 8, pp. 65060-65070, 2020, doi: 10.1109/ACCESS.2020.2985717

9. Mallick S. Challenges of mainstreaming: Ayurvedic practice in Delhi Government health institutions. Journal of Ayurveda and integrative medicine. 2016 Mar 1;7(1):57-61. https://doi.org/ 10.1016/j.jaim.2015.10.001

10. Patwardhan B. (2016). Strengthening the Ayurveda ecosystem. Journal of Ayurveda and integrative medicine, 2016 Apr;7(2):73.. https:// doi.org/10.1016/j.jaim.2016.07.002

11. Kessler CS, Morandi A, Kumar A, Dhiman KS, Gupta S, Icke K, Bühner C, Stapelfeldt E, Wischnewsky M, Kronpaß L, Murthy V, Michalsen A, Witt CM. Reliability of Ayurvedic Diagnosis for Knee Osteoarthritis Patients: A Nested Diagnostic Study Within a Randomized Controlled Trial. J Altern Complement Med. 2019 Sep;25(9):910-919. doi: 10.1089/ acm.2018.0273. Epub 2019 Jan 17. PMID: 30653338 ; PMCID: PMC6748397.

12. Banerjee S, Biswas TK, Chattopadhyay K, Arzoo SH, Chattopadhyay B. An Approach to Screen Genotoxic-Susceptible Diabetic Population of Various Prakriti Groups for Personalized Disease Management. J Altern Complement Med. 2020 Oct 19. https://doi.org/10.1089/acm.2020.0001

13. Patwardhan B, Bodeker G. Ayurvedic genomics: establishing a genetic basis for mind-body typologies. J Altern Complement Med. 2008 Jun 1;14(5):571-6. https://doi.org/10.1089/ acm.2007.0515

14. Rastogi S. Development and validation of a Prototype Prakriti Analysis Tool (PPAT): Inferences from a pilot study. Ayu. 2012 A p r ; 3 3 ( 2): $209-18$. d o i : 10.4103/0974-8520.105240

15. Rapolu SB, Kumar M, Singh G, Patwardhan K. Physiological variations in the autonomic responses may be related to the constitutional types defined in Ayurveda. Tang [Internet]. 2015 Feb 28;5(1):7.1-7.7. Available from: https:// doi.org/10.5667/TANG.2014.0031

16. Agrawal S, Gehlot S. DO THE FUNCTIONS OF PITTA DOSHA SUBTYPES VARY AS PER PRAKRITI-A CROSS SECTIONAL STUDY. Journal of Research and Education in Indian Medicine. 2018;24(1):51-7. http://dx.doi.org/ 10.5455/JREIM.82-1506163089

17. Rotti H, Raval R, Anchan S, et al. Determinants of prakriti, the human constitution types of Indian traditional medicine and its correlation with contemporary science. Journal of Ayurveda and Integrative Medicine. 2014 Jul;5(3):167-175. DOI: $10.4103 / 0975-9476.140478$.

18. Chatterjee, Bijoya, and Jigisha Pancholi. "Prakriti-Based Medicine: A Step towards Personalized Medicine." Ayu 32, no. 2 (April 2011): 141-46. https://doi .org/ 10.4103/0974-8520.92539.

19. Hankey A. A test of the systems analysis underlying the scientific theory of Ayurveda's Tridosha. J Altern Complement Med. 2005 Jun;11(3):385-90. doi: 10.1089/acm.2005.11.385. PMID: 15992219.

20. Lakhotia SC. Translating Ayurveda's DoshaPrakriti into objective parameters. J Ayurveda Integr Med. 2014 Jul;5(3):176. PMID: 25336849; PMCID: PMC4204288.

21. Sharma H, Keith Wallace R. Ayurveda and Epigenetics. Medicina (Kaunas). 2020 Dec 11;56(12):687. doi: 10.3390/medicina56120687. PMID: 33322263; PMCID: PMC7763202.

22. Hankey, Alex. "Establishing the Scientific Validity of Tridosha Part 1: Doshas, Subdoshas and Dosha Prakritis." Ancient Science of Life 29, no. 3 (January 2010): 6-18.

23. Center for development of advanced Computing, [Home page on internet] Ayusoft, https:// w w w . c d a c . i n / i n d ex. a s p x ? id=hi_dss_prakriti_vichaya. Last accessed on 31-01-2021.

24. Bhalerao S, Deshpande T, Thatte U. Prakriti (Ayurvedic concept of constitution) and variations in platelet aggregation. BMC Complement Altern Med. 2012;12:248. https:// doi.org/10.1186/1472-6882-12-248.

25. Tripathi PK, Patwardhan K, Singh G. The basic cardiovascular responses to postural changes, exercise, and cold pressor test: do they vary in accordance with the dual constitutional types of ayurveda?. Evidence-Based Complement and Alternative Medicine. 2011 Jan 1;2011. https:// doi.org/10.1155/2011/251850.

26. Streiner DL, Norman GR, Cairney J. Health Measurement Scales: A practical guide to their development and use [Internet]. Health Measurement Scales. Oxford University Press; [cited 2021 Jan 31]. Available from: https:// oxfordmedicine.com/view/10.1093/med/ 9780199685219.001.0001/med-9780199685219. 


\section{Annexure - New tool for prakriti assessment}

\begin{tabular}{|c|c|c|c|c|c|c|c|}
\hline \multicolumn{8}{|c|}{ Grading: 0-Absent; 1-Doubtful; 2-Evident; 3-Clearly evident } \\
\hline Sl. No. & Feature & Vata & Grade & Pitta & Grade & Kapha & Grade \\
\hline 1 & Skin & Dry & A1 & Profuse sweating type & B1 & Moist/smooth & $\mathrm{C} 1$ \\
\hline 2 & Eyes & Round, Lusterless (unclear) & A2 & Turns red easily & B2 & $\begin{array}{c}\text { Wide and elongated } \\
\text { with plenty of eye } \\
\text { lashes }\end{array}$ & $\mathrm{C} 2$ \\
\hline 3 & $\begin{array}{l}\text { Sound/ } \\
\text { speech }\end{array}$ & Talkative, irrelevant talks & A3 & Rough when angry & B3 & $\begin{array}{l}\text { Relevant \& Soft } \\
\text { spoken }\end{array}$ & $\mathrm{C} 3$ \\
\hline 4 & Appetite & $\begin{array}{l}\text { Severe at times, diminished } \\
\text { at times }\end{array}$ & A4 & $\begin{array}{l}\text { Strong appetite and } \\
\text { digestive capacity, } \\
\text { Intolerance to hunger }\end{array}$ & B4 & $\begin{array}{l}\text { Generally reduced } \\
\text { appetite, hunger can be } \\
\text { tolerated }\end{array}$ & $\mathrm{C} 4$ \\
\hline 5 & Sleep & Wakes up intermittently & A5 & Moderate sleep & B5 & Deep sleep & $\mathrm{C} 5$ \\
\hline 6 & $\begin{array}{l}\text { Memory/ } \\
\text { intellect }\end{array}$ & Grasps easily, forgets easily & A6 & $\begin{array}{l}\text { Curious in new matters } \\
\text { and ideas }\end{array}$ & B6 & $\begin{array}{l}\text { Grasps slowly but } \\
\text { good memory }\end{array}$ & C6 \\
\hline 7 & Interest & $\begin{array}{l}\text { Hot(1), sweet(2), } \operatorname{sour}(3), \\
\text { salt( } 4)\end{array}$ & A7 & $\begin{array}{l}\text { Cold(1), sweet(2), } \\
\text { bitter(3) }\end{array}$ & B7 & $\begin{array}{l}\text { Hot(1), bitter(2), } \\
\text { pungent(3), } \\
\text { astringent(4) }\end{array}$ & $\mathrm{C} 7$ \\
\hline 8 & Friendship & $\begin{array}{l}\text { Few friends, unsteady } \\
\text { company }\end{array}$ & A8 & $\begin{array}{l}\text { Becomes support for } \\
\text { the dependents }\end{array}$ & B8 & Strong & $\mathrm{C} 8$ \\
\hline & Score & $\begin{array}{ll}\mathrm{V} \\
\end{array}$ & & $\begin{array}{l}\mathrm{P} \\
\end{array}$ & & $\mathbf{K}$ & \\
\hline \multicolumn{8}{|c|}{$\mathrm{V}=\mathrm{A} 1 * 5+\mathrm{A} 2 * 3+\mathrm{A} 3 * 6+\mathrm{A} 4 * 5+\mathrm{A} 5 * 4+\mathrm{A} 6 * 5+\mathrm{A} 7(1) * 7+\mathrm{A} 7(2) * 5+\mathrm{A} 8 * 5$} \\
\hline \multicolumn{8}{|c|}{$\mathrm{P}=\mathrm{B} 1 * 8+\mathrm{B} 2 * 3+\mathrm{B} 3 * 5+\mathrm{B} 4 * 7+\mathrm{B} 5 * 4+\mathrm{B} 6 * 5+\mathrm{B} 7(1) * 5+\mathrm{B} 7(2) * 3+\mathrm{B} 7(3) * 3+\mathrm{B} 8 * 3$} \\
\hline \multicolumn{8}{|c|}{$\mathrm{K}=\mathrm{C} 1 * 4+\mathrm{C} 2 * 4+\mathrm{C} 3 * 6+\mathrm{C} 4 * 5+\mathrm{C} 5 * 6+\mathrm{C} 6 * 5+\mathrm{C} 7(3) * 7+\mathrm{C} 7(4) * 5+\mathrm{C} 8 * 5$} \\
\hline \multicolumn{8}{|c|}{$\mathrm{VP}=\mathrm{B} 1 * 2+\mathrm{A} 3 * 4+\mathrm{B} 3 * 2+\mathrm{A} 4 * 2+\mathrm{B} 4 * 2+\mathrm{A} 5 * 2+\mathrm{B} 5 * 2+\mathrm{A} 6 * 3+\mathrm{B} 6+\mathrm{A} 7(2) * 5+\mathrm{A} 8$} \\
\hline \multicolumn{8}{|c|}{$\mathrm{VK}=\mathrm{A} 1 * 2+\mathrm{C} 1+\mathrm{C} 3+\mathrm{A} 4 * 2+\mathrm{C} 4 * 2+\mathrm{C} 5 * 2+\mathrm{C} 6+\mathrm{A} 7(1) * 7+\mathrm{A} 8 * 2+\mathrm{C} 8$} \\
\hline \multicolumn{8}{|c|}{$\mathrm{KP}=\mathrm{B} 1 * 3+\mathrm{C} 1 * 3+\mathrm{C} 2 * 2+\mathrm{C} 3 * 3+\mathrm{B} 4+\mathrm{C} 4+\mathrm{B} 5+\mathrm{C} 5 * 2+\mathrm{B} 6 * 2+\mathrm{C} 6+\mathrm{B} 7(2) * 3+\mathrm{C} 7(4) * 3+\mathrm{B} 8 * 2+\mathrm{C} 8 * 3$} \\
\hline \multicolumn{8}{|c|}{ Final Diagnosis } \\
\hline Prakriti & & & & & & & \\
\hline
\end{tabular}

